\title{
Single-Electrode-Based Rotatinary Triboelectric Nanogenerator and its Applications as Self-Powered Contact Area and Eccentric Angle Sensors
}

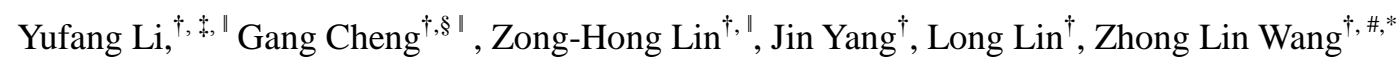

${ }^{\dagger}$ School of Material Science and Engineering, Georgia Institute of Technology, Atlanta, Georgia 30332-0245, United States

${ }^{\$}$ School of Material Science and Technology, Nanjing University of Aeronautics and Astronautics, Nanjing, China

${ }^{\S}$ Key Lab for Special Functional Materials, Henan University, Kaifeng, 475004, China

${ }^{\#}$ Beijing Institute of Nanoenergy and Nanosystems, Chinese Academy, Chinese Academy of Sciences, China

* To whom correspondence should be addressed: zlwang@gatech.edu

${ }^{\|}$Y. Li, G. Cheng and Z.-H. Lin contributed equally to this work.

\section{Abstract:}

We introduce a single-electrode-based rotationary triboelectric nanogenerator (SR-TENG) formed by two wheels and a belt for harvesting mechanical energy. The fundamental working principle is studied by conjunction of experimental results with finite element calculation. The continuous discharging (CD) mode and the instantaneous discharging (ID) mode have been demonstrated for the SR-TENG. The systematical experiments indicate that the short-circuit current increases with the rotating speed for SR-TENG with CD mode, but the open-circuit voltage maintains constant. The short-circuit current and open-circuit voltage decrease nearly linearly with the friction contact area, which provides an application as a self-powered surface area sensor of transmission wheel and gear. For SR-TENG with ID mode, the electric outputs are greatly enhances. The current peak is about $20 \mu \mathrm{A}$ at variation rotating speeds even if the external load is $10 \mathrm{M} \Omega$, which is 33 times higher than that of the SR-TENG with CD mode 
without external load. The SR-TENG with ID mode has also been demonstrated as a selfpowered misalignment sensor.

Keywords: triboelectric nanogenerator, rotating energy, continuous discharge, instantaneous discharge, self-powered sensor 


\section{Introduction}

Over the past half century, the development of electronic devices is toward miniaturization, lightweight, and portable. In addition, the worldwide energy consumption is growing rapidly. Therefore, new technologies that can harvest energy from the environment as sustainable and low-cost power source are highly desirable. ${ }^{[1,2]}$ There are various forms of mechanical energy existed in our living environment, such as rotation, vibration, walking and so on. Many ways to harvest mechanical energy has been developed that are based on electromagnetics, ${ }^{[3,4]}$ piezoelectrics, ${ }^{[5-9]}$ and electrostatics ${ }^{[10,11]}$ effects, which have been extensively developed for decades. Recently, triboelectric nanogenerator (TENG) ${ }^{[12-21]}$ has been developed based on the universally known triboelectric effect as a cost-effective and robust energy technology, which can efficiently convert mechanical energy into electricity for self-powered applications without reliance on traditional power supplies. Various applications have been demonstrated, such as micropatterning, ${ }^{[22]}$ powering portable electronics ${ }^{[23-25]}$ and self-powered sensors. ${ }^{[26,27]}$ In all kinds of TENGs, the rotating TENG can harvest rotational energy through a periodic in-plane sliding displacement between two friction surfaces. ${ }^{[18,21,28]}$ While, these rotating TENG also has limitations for application, for example, it is needed to be fixed on a rotating machine. In practical applications, some rotating machines consist of two rotating wheels and belt, such as transfer machine and engine. By combining the inherent structure of these rotating machines and the working mechanism of rotating TENG, novel TENG and self-powered sensors can be developed.

In this paper, we developed a single-electrode-based rotating (SR)-TENG which contains two rotatable wheels and a polytetrafluoroethene (PTFE) belt. Two working modes, the continuous discharged (CD) mode and the instantaneous discharged (ID) mode have been demonstrated for the SR-TENG. For SR-TENG with CD mode, the short-circuit current and open-circuit voltage decrease nearly linearly with the friction contact area, which provides an 
application as a self-powered surface area sensor of transmission wheel and gear. For SRTENG with ID mode, the instantaneous electric outputs are greatly enhances. The SR-TENG with ID mode has also been demonstrated as a self-powered misalignment sensor. This study extends the potential application of SR-TENG as multi-functional self-powered sensors.

\section{Experimental Section}

\section{A. Fabrication of the Nanostructured PTFE Film}

First, microstructures were fabricated by blasting an $\mathrm{Al}$ foil with sand particles using compressed air. The sand-blasted Al foil was further anodizing in a 0.3 Moxalic acid solution to obtain an anodic $\mathrm{Al}$ oxide (AAO) template with nanometer-sized holes, and the average diameter of the holes in the AAO template is about $42 \mathrm{~nm}$. Then the PTFE solution was poured into the AAO template and a conventional vacuum process was applied to remove the air remaining in the nanoholes. After the curing at ambient temperature for one day, the solvent was evaporated and leaved a PTFE thin film with nanostructures. Finally, the PTFE thin film was peeled off from the AAO template using a double-sided tape.

\section{B. Fabrication of the TENG}

First, Four 1"-thick PMMA sheets were processed by laser cutting (PLS6.75, Universal Laser Systems) to form the two wheels. One wheel was connected to the rotational motor. On one wheel surface, a layer of PDMS is coated, and the elastic property of PDMS enables a complete contact of two tribo-surfaces. On the top of PDMS layer, half of the wheel is covered by Al film and half overlay is PTFE film. The wheel with PDMS is rotatable wheel and a PTFE belt. The PTFE belt acts as a triboelectric polymer, while the Al film plays dual roles as a triboelectric layer and an electrode.

\section{Electric Output Measurement of TENG}

In the electric output measurement of the TENG, the current meter (SR570 low noise current amplifier, Stanford Research System) and voltage meter (6514 system electrometer, 
Keithley) were used to measure the electric outputs of the TENG.

\section{Results and discussion}

The main structure of the SR-TENG is composed of two rotatable wheels (with a same radius of $5 \mathrm{~cm}$ ) and a PTFE belt (with a width of $5 \mathrm{~cm}$ ), which schematically illustrated in Figure 1a. In the fabrication of the SR-TENG, poly (methyl methacrylate) (PMMA) are chosen as wheel materials to construct the device because of its low cost, light weight, decent strength, and easy processing. On one wheel surface, a layer of polydimethylsiloxane (PDMS) is coated. The presence of PDMS enables a more effective contact of two tribo-surfaces during the operating process. Then, half of the PDMS layer is covered by an Al film and the other half of the PDMS is covered by a PTFE film (Figure 1a). The Al film here will play dual roles as a triboelectric contact material and a conductive electrode, while the other triboelectric contact material is the PTFE film. In order to increase the effective contact area of the two tribo-surfaces, and enhance the electrical output of the SR-TENG, the inner surface of the PTFE belt was modified with dense nanorods (Figure 1b). The SEM image displays that the average diameter of the PTFE nanorods is $45 \mathrm{~nm}$ (Figure 1c).

The working principle of the SR-TENG can be explained by the contact-induced electrification and the variation of induction charges caused by relative sliding between the wheels and the PTFE belt. During their relative rotating process, the contact electrification will cause the inner surface of PTFE with negative tribo-charges, and the Al film surface and PMMA surface with positive tribo-charges. ${ }^{[2]}$ The diagrams of charge distribution and current generation at four different rotating angles $\left(0^{\circ}, 90^{\circ}, 180^{\circ}\right.$, and $\left.270^{\circ}\right)$ are shown in Figure $1 \mathrm{~d}-1 \mathrm{~g}$. Before the rotation starts, the rotating angle is $0^{\circ}$ (the $\mathrm{Al}$ film is completely overlapping with the PTFE belt), and the induced charge density on Al film surface reaches a maximum value (Figure 1d). When the rotating angle is increased to $90^{\circ}$ (Figure 1e), the $\mathrm{Al}$ film and PTFE belt become partially separated, which induces the $\mathrm{Al}$ film with a higher potential than the ground. 
The electrons will flow from the ground to the $\mathrm{Al}$ film to decrease the potential of the $\mathrm{Al}$ film to zero. As the $\mathrm{Al}$ film is completely separated from the PTFE belt (the rotating angle is $180^{\circ}$ ), the induced charge density on Al film surface decreases to a minimum value (Figure 1f). When the wheel continues to rotate (the rotating angle is $270^{\circ}$ ) and the Al film surface contacts with the PTFE belt again, a lower potential of the Al film than the ground is formed. As a result, the electrons will flow from the $\mathrm{Al}$ film to the ground (Figure 1g). As the rotation continues, the Al film and the PTFE belt will be fully contacted again (Figure 1d) and then another new cycle (from Figure 1d to Figure 1g) begins. Since the induced charges are continuously charged/discharged during the rotating process, this working mode will be defined as the continuous discharging (CD) mode ${ }^{[29]}$. As shown in Figure 1h, another device structure is also designed, in which both wheels are covered with PDMS, Al film and PTFE film.

To obtain a more quantitative understanding of the proposed working principle, a numerical simulation via finite element method (FEM) has been employed to calculate the potential distribution in open-circuit condition and the change of induced charge density on the Al film surface in short-circuit condition. The model using here has the same structure and dimension as the real device. The triboelectric charge density on the inner surface of PTFE belt was assigned to be $60 \mu \mathrm{C} / \mathrm{m}^{2}$. Figure $2 \mathrm{a}-2 \mathrm{f}$ shows the calculated potential distribution in a full cycle of rotation $\left(360^{\circ}\right)$ with a step of $60^{\circ}$. When the Al film and PTFE belt are fully contacted (the rotation angle is $0^{\circ}$ ), the potential on the $\mathrm{Al}$ film surface is the highest. Subsequently, as the Al film begins to rotate, the potential on the Al film surface decreases gradually (Figure $2 \mathrm{~b}$ and $2 \mathrm{c}$ ). As the rotation angle is $180^{\circ}$ (Figure $2 \mathrm{~d}$ ), the potential on the $\mathrm{Al}$ film surface decreases to a minimum value. Rotating angle between $0^{\circ}$ and $180^{\circ}$ is the first half working cycle of the SR-TENG. With the rotating angle continuously increasing, the other half working cycle of the SR-TENG starts. The potential on the Al film surface becomes larger as the contact area between the Al film and PTFE belt increases. The dependence of the 
open-circuit voltage $\left(\mathrm{V}_{\mathrm{oc}}\right)$ to-ground of the $\mathrm{Al}$ film on rotating angle is plotted in Figure $2 \mathrm{~g}$, in which the $\mathrm{V}_{\text {oc }}$ varies with the rotating angle in a parabola shape. These results fully support the working principle we proposed in Figure 1. In the short-circuit condition, the dependence of the induced charge density of the Al film on rotating angle is shown in Figure $2 \mathrm{~h}$, in which the charges density decreases with the increase of rotating angle from $0^{\circ}$ to $180^{\circ}$. Then as the rotating angle increases from $180^{\circ}$ to $360^{\circ}$, the induced charge density of the Al film will also increase. The charges periodically transfer between the $\mathrm{Al}$ film and the ground when the wheel rotates. The maximum transferred charge density can be up to $16.14 \mu \mathrm{C} / \mathrm{m}^{2}$. Figure $2 \mathrm{i}$ shows the calculated current density in a full rotation cycle with a rotating speed of $300 \mathrm{rpm}$. It can be observed that the current density curve displays an AC form, which is confirmed by the experimental results (Figure 3).

To characterize the electrical output, the short-circuit current ( $\left.I_{s c}\right)$ and $V_{o c}$ curves of the SR-TENG with CD mode were measured. Figure $3 \mathrm{a}$ shows the measured $\mathrm{I}_{\mathrm{sc}}$ at a rotating speed of $300 \mathrm{rpm}$, and the inset is the magnification of the current curve. The $\mathrm{V}_{\mathrm{oc}}$ reached a value of $60 \mathrm{~V}$ (from $-20 \mathrm{~V}$ to $+40 \mathrm{~V}$ ). To investigate the influence of rotating speed on the output performance of the SR-TENG, the $\mathrm{I}_{\mathrm{sc}}$ at different rotating speed were measured, which reveal that the $I_{\mathrm{sc}}$ becomes larger with increasing the rotating speed from 100 to $1100 \mathrm{rpm}$ (Figure 3c). There is an approximately linear relationship between the rotating speed and the $\mathrm{I}_{\mathrm{sc}}$. These results provide a reliable application of the SR-TENG as a self-powered rotating speed sensor. Figure $3 \mathrm{~d}$ gives the $\mathrm{V}_{\mathrm{oc}}$ at different rotating speed, in which the $\mathrm{V}_{\mathrm{oc}}$ almost keep constant even increasing the rotating speed, because the voltage is only a function of rotating angle, as discussed in Figure $2 \mathrm{~g}$. This result consists well with the previous results [21, 27].

For TENG, the output will varies when connecting to the external loads with different resistances [2]. This is a critical issue when we apply the TENG to directly harvest energy from the environment. In this regard, the output performance of the SR-TENG was systematically studied at different loads. Figure 3e displays the dependence of instantaneous 
output current and power on different load resistances (from $10 \mathrm{~K} \Omega$ to $1 \mathrm{G} \Omega$ ) at a rotating speed of $500 \mathrm{rpm}$. It is clearly shown that the output current maintains a constant value when the resistances are below $10 \mathrm{M} \Omega$, then decreases with the increasing of resistance. The output power shows a positive trend as the resistance increases at a low resistance region (from 10 $\mathrm{K} \Omega$ to $250 \mathrm{M} \Omega$ ), and then decreases with the increasing of resistance between $250 \mathrm{M} \Omega-1 \mathrm{G} \Omega$. The maximum instantaneous power value is $\sim 8.2 \mu \mathrm{W}$ at a resistance of $250 \mathrm{M} \Omega$. It can be found in Figure S1 that the power peak value increases with the increment of rotating speed at a fixed external load of $10 \mathrm{M} \Omega$. The power peak value is almost linearly with the rotating speed. To demonstrate the SR-TENG can be used as a direct power source to drive the electronics, 20 serial-connected commercial LEDs were connected to the output of the SRTENG. As shown in Figure 3f, 20 LEDs were powered by the SR-TENG when the rotating speed is $1100 \mathrm{rpm}$. Moreover, the LEDs can be powered continuously rather than just flashing. As a power source, the long-term stability of the electricity generated by the TENG is needed for practical application. As shown in Figure S2, the short-circuit current is stable during a period of 45 minutes as the TENG operates at a rotating speed of $500 \mathrm{rpm}$.

The concept of the SR-TENG can be also used to detect the contact area change between the wheels and belt. For the demonstration, different sizes of the Al film were applied and the electrical outputs were measured. As shown in Figure 4a, an Al film with dimensions of 10 $\mathrm{mm} \times 50 \mathrm{~mm}$ was used. Then we fixed the width $(10 \mathrm{~mm})$ of the Al film and only changed the length (h) (50mm) of the $\mathrm{Al}$ film to $45,40,35$, and $30 \mathrm{~mm}$, respectively. In other words, the effective contact area of the Al film will loses 10\%, 20\%, 30\% and 40\%, respectively. The electrical output of the SR-TENG with different sized Al films operating at a rotating speed of $500 \mathrm{rpm}$ was shown in Figure $4 \mathrm{~b}$. It is observed that both the $\mathrm{V}_{\mathrm{oc}}$ and $\mathrm{I}_{\mathrm{sc}}$ decreases from $19 \mathrm{~V}$ to $9 \mathrm{~V}$ and $0.4 \mu \mathrm{A}$ to $0.25 \mu \mathrm{A}$, respectively, as the contact area reduces from $10 \times 50 \mathrm{~mm}^{2}$ to $10 \times 30 \mathrm{~mm}^{2}$. This is because reducing the contact area will decrease the transferred charges, therefore affecting the electrical output of the SR-TENG (Figure 4d). This demonstration is 
very important. Currently for metal bodies such as bearings, gears, wheel/rail, the contact damage of online monitoring is built on sound or vibration abrasive as a failure warning or diagnosis. However, in the actual work of even large contact surface pitting and coating peeling off, those approaches will not give the diagnosis just a short time before total failure, which often leads to institution of abnormal shutdowns and unscheduled maintenance. As shown in Figure 4, the electrical outputs changes with area, based on which a reliable approach of employing the SR-TENG as a self-powered surface area sensor of transmission wheel and gear can be developed. When rolling surface has some defects, such as cracks, pits, spalls and oxide inclusion, the contact area will be reduced. Therefore, the output current and voltage will decrease with the increasing defects. It can be realized by using the output signal of current or voltage to acquire the information of surface defects for rolling wheel or gear. It is a useful tool for early detection and progressive monitoring of components deterioration.

As mentioned above, the electrode is directly connecting to the ground with metal wires, and the SR-TENG works at CD mode. In the following, another working mode, instantaneous discharging (ID) mode ${ }^{[29]}$, was demonstrated to improve the instantaneous electrical output of SR-TENG. The device structure of the ID mode-based SR-TENG is shown in Figure 5a. A needle-shaped $\mathrm{Al}$ electrode $\left(\mathrm{K}_{0}\right)$ is fixed at one end of $\mathrm{Al}$ film on the wheel, and connected with the $\mathrm{Al}$ film. A thin $\mathrm{Al}$ bar is connected with the ground, which can be divided into two parts: top part $\left(\mathrm{K}_{1}\right)$ and bottom part $\left(\mathrm{K}_{2}\right)$. During the rotating process, the $\mathrm{Al}$ film is connected to the ground only when it is fully contacted with the PTFE belt ( $\mathrm{K}_{0}$ and $\mathrm{K}_{1}$ are closed) or it is fully separated with the PTFE belt ( $\mathrm{K}_{0}$ and $\mathrm{K}_{2}$ are closed), and the Al film is disconnected with the ground in other time. Figures $5 \mathrm{~b}-5 \mathrm{e}$ show the working mechanism of the SR-TENG with ID mode. The $\mathrm{K}_{0}, \mathrm{~K}_{1}$ and $\mathrm{K}_{2}$ constitute a trigger switch. At the initial state (Figure $5 \mathrm{~b}$ ), the Al film is fully contacted with the PTFE. At this moment, $\mathrm{K}_{0}$ and $\mathrm{K}_{1}$ are closed, therefore, the positive charges can flow from the ground the $\mathrm{Al}$ film to reach electric equilibrium. As the wheel rotates, the switch is open (Figure 5c), and the charges on the Al film remain the same 
because the electrode is disconnected with the ground. At the stage of Figure 5d, the Al film is fully separated with the PTFE film, and the switch is closed again. Since the potential of Al film is higher than the ground, the electrons are driven to flow instantaneously form the ground to $\mathrm{Al}$ film. With the further rotating of the wheel, the switch remains open, and there are no charges on the $\mathrm{Al}$ film, as shown in Figure 5e. As the Al film is fully contacted with the PTFE belt, the switch is closed again (Figure 5b), and a new cycle starts. As a result, only two pulsed electrical peaks are generated during a rotating cycle.

The output current and voltage of the SR-TENG operating with ID mode were measured at different rotating speeds. Figure $5 \mathrm{f}$ shows an external load of more than $1 \mathrm{M} \Omega$ needs to be serially connected to the circuit of the SR-TENG with ID mode, because of the limitation of the range and bandwidth of the current meter $(5 \mathrm{~mA}, 1 \mathrm{MHz})$ used in experiment. The current of SR-TENG with ID mode with a load of $10 \mathrm{M} \Omega$ measured at a rotating speed of $300 \mathrm{rpm}$ is given in Figure 5f, with the insert of magnification of a single current peak. Even if the external load is $10 \mathrm{M} \Omega$, the current peak of the SR-TENG with ID mode is about $20 \mu \mathrm{A}$, which is 33 times higher than that of the SR-TENG with CD mode (Figure 3a) without external load at the same rotating speed. From the magnification of a single current peak of the SR-TENG with ID mode, it can be found that the current increases abruptly from zero to its peak and then decreases quickly from peak to zero, instead of slowly raising and lowering like SR-TENG with CD mode (Figure 3a). All these results show that current is greatly enhanced by the SR-TENG with ID mode. Figures $5 \mathrm{~g}$ and $5 \mathrm{~h}$ give the dependences of the output current and voltage peaks on rotating speeds ranging from 100 to $900 \mathrm{rpm}$, respectively. The current peaks and voltage peaks almost stay constants around $20 \mu \mathrm{A}$ and $70 \mathrm{~V}$ at various rotating speeds, respectively, which illustrated that the contact-separation speed between two tribo-surfaces does not influence the current and voltage of the SR-TENG with ID mode.

From those results, it is clear that the SR-TENG with ID mode has a much faster charging/discharging speed than that in the $\mathrm{CD}$ mode. For the $\mathrm{CD}$ mode, this speed is 
limited by the mechanical contact-separation speed between the two tribosurfaces, which depends on rotating speed. This speed is not limited by the rotating speed for the ID mode, and the inductive charges can be instantaneously charged/discharged as the needle-shape $\mathrm{Al}$ electrode is contacted with the top part or bottom part of the grounded Al electrode (Figure 5a), which is the essential reason for the enhancement of the current.

Shaft eccentricity is an important parameter for motors with high rotating speed. Considerable damage will happen if eccentric shaft rotates at very high speed and very high amplitude. The SR-TENG with ID mode can be used as a self-powered sensor of motor shaft eccentric angle according to its instantaneous discharge characteristic. Figure $6 \mathrm{a}$ shows the diagram of the self-powered sensor, in which a metal central shaft is electrically connected with the $\mathrm{Al}$ film, a grounded $\mathrm{Cu}$ rod is placed below the shaft, the perpendicular distance between the shaft and the rod is expressed as $\mathrm{d}$, and the perpendicular distance between the rod and the rotating wheel is expressed as L. If there is eccentric angle of the rotating shaft, the moving trace of the shaft is not a line but a cone shape, as shown in inset of Figure 6b. For a certain L value and eccentric angle $(\Theta)$, there is a critical distance $\left(\mathrm{d}_{0}\right)$. The instantaneous current signal will be generated when $d$ is less than $d_{0}$. The relation between $\Theta$, L, and $\mathrm{d} 0$ can be expressed as:

$$
\operatorname{tg} \theta=\frac{d_{0}}{L}
$$

In real applications, the centrifugal angle can be derived by measuring the $\mathrm{L}$, and $\mathrm{d}_{0}$ values. For an $\mathrm{L}$ value of $60 \mathrm{~mm}$, the distance $\mathrm{d}$ is changed from $10 \mathrm{~mm}$ to $2 \mathrm{~mm}$, in other words, the copper rod gradually close to the shaft. The output current curves at different $d$ values at a rotating speed of $500 \mathrm{rpm}$ and a load of $10 \mathrm{M} \Omega$ are shown in Figure $6 \mathrm{~b}$, and the dependence of the current peaks on $d$ is plotted in Figure $6 \mathrm{c}$. When $\mathrm{d}$ is larger than $4 \mathrm{~mm}$, the current sharply decreases to nearly zero, from which it can be obtained that $\mathrm{d} 0$ is $4 \mathrm{~mm}$. According to Equation (1), the centrifugal angle of the wheel is calculated as $3.67^{\circ}$.In order to reduce the 
measurement errors, the $d_{0}$ values at various $L$ values were measured, and their relationship is plotted in Figure 6d, which can be fitted by a straight line. By calculating the slope of the fitted line, it can be derived that the $\Theta$ value is 3.78

\section{Conclusion}

In summary, we demonstrated a SR-TENG based on sliding electrification for converting rotational mechanical energy into electricity. Finite element simulation was employed to gain a comprehensive understanding about the fundamental working principle. For SR-TENG with $\mathrm{CD}$ mode, there is an approximately linear relationship between the rotating speed and the current, which provide a reliable approach of employing the TENG as a self-powered velocity sensor. Through studying the effects of tiny changes in the friction contact area on the output performance, the short-circuit current and open-circuit voltage decrease nearly linearly with the area reduced ratio. This is a reliable approach of employing the SR-TENG as a selfpowered surface area sensor of transmission wheel and gear. By using a contact switch, we also have developed an SR-TENG with ID mode that greatly enhances the instantaneous electric outputs of the TENG. The current peak of the SR-TENG with ID mode is about 20 $\mu \mathrm{A}$ even if the external load is $10 \mathrm{M} \Omega$, which is 33 times higher than the short-circuit current peak of the SR-TENG in the CD mode. In addition, the SR-TENG with ID mode is demonstrated as a self-powered sensor for detecting centrifugal angle of a rotating wheel.

\section{Acknowledgements}

Research was supported by U.S. Department of Energy, Office of Basic Energy Sciences (award DE-FG02-07ER46394), and the "Thousands Talents" Program for Pioneer Researcher and His Innovation Team, China, Beijing City Committee of Science and Technology 
(projects Z131100006013004, Z131100006013005). Patents have been filed based on the research presented here.

\section{Appendix A. Supplementary Material}

Supplementary data associated with this article can be found in the online version.

\section{References}

[1] Z. L. Wang, G. Zhu, Y. Yang, C. F. Pan, Materials Today. 15 (2012) 532-543.

[2] Z. L. Wang, ACS nano. 7 (2013) 9533-9557.

[3] C. B. Williams, C. Shearwood, M. A. Harradine, P. H. Mellor, T. S. Birch, R. B. Yates, Proc. IEEE Circ. Dev. Syst. 148 (2001) 337-342.

[4] S. P. Beeby, R. N. Torah, M. J. Tudor, P. Glynne-Jones, T. O'Donnell, C. R. Saha, S. Roy, J. Micromech. Microeng. 17 (2007) 1257-1265.

[5] Z. L. Wang, J. H. Song, Science. 312 (2006) 242-246.

[6] Y. Qin, X. D. Wang, Z. L. Wang, Nature. 451 (2008) 809-813.

[7] C. E. Chang, V. H. Tran, J. B. Wang, Y. K. Fuh, L. W. Lin, Nano Lett. 10 (2010) 726-731.

[8] X. Chen, S. Y. Xu, N. Yao, Y. Shi, Nano Lett. 10 (2010) 2133-2137.

[9] Y. F. Hu, L. Lin, Y. Zhang, Z. L. Wang, Adv. Mater. 24 (2012) 110-114.

[10] P. D. Mitcheson, P. Miao, B. H. Stark, E. M. Yeatman, A. S. Holmes, T. C. Green, Sens. Actuators A. 115 (2004) 523-529.

[11] Y. Naruse, N. Matsubara, K. Mabuchi, M. Izumi, S. Suzuki, J. Micromech. Microeng. 19 (2009) 094002.

[12] F. R. Fan, Z. Q. Tian, Z. L. Wang, Nano Energy. 1 (2012) 328-334.

[13] F. R. Fan, L. Lin, G. Zhu, W. Z. Wu, R. Zhang, Z. L. Wang, Nano Lett. 12 (2012) 3109-3114.

[14] G. Zhu, C. F. Pan, W. X. Guo, C. Y. Chen, Y. S. Zhou, R. M. Yu, Z. L. Wang, Nano Lett. 12 (2012) 4960-4965.

[15] S. H. Wang, L. Lin, Z. L. Wang, Nano Lett. 12 (2012) 6339-6346.

[16] X. S. Zhang, M. D. Han, R. X. Wang, F. Y. Zhu, Z. H. Li, W. Wang, H. X. Zhang, Nano Lett. 13 (2013) $1168-1172$.

[17] G. Zhu, Z.-H. Lin, Q. S. Jing, P. Bai, C. F. Pan, Y. Yang, Y. S. Zhou, Z. L. Wang, Nano Lett. 13 (2013) 
847-853.

[18] Y. Yang, L. Lin, Y. Zhang, Q. S. Jing, T. C. Hou, Z. L. Wang, ACS Nano. 6 (2012) 10378-10383.

[19] S. H. Wang, L. Lin, Y. N. Xie, Q. S. Jing, S. M. Niu, Z. L. Wang, Nano Lett. 13 (2013) 2220-2225.

[20] G. Zhu, J. Chen, Y. Liu, P. Bai, Y. S. Zhou, Q. S. Jing, C. F. Pan, Z. L. Wang, Nano Lett. 13 (2013) 2282-2289.

[21] L. Lin, S. H. Wang, Y. N. Xie, Q. S. Jing, S. M. Niu, Y. F. Hu, Z. L. Wang, Nano Lett. 13 (2013) 29162923.

[22] G. Zhu, C. Pan, W. Guo, C.-Y. Chen, R. Yu, Z. L. Wang, Nano Lett. 12 (2012) 4960-4965.

[23] X. Y. Xue, P. Deng, B. He, Y. X. Nie, L. Xing, Y. Zhang, Z. L. Wang, Adv. Energy Mater. 4 (2014) 1301329

[24] P. Bai, G. Zhu, Z.-H. Lin, Q. Jing, J. Chen, G. Zhang, J. Ma, Z. L. Wang, ACS Nano. 7 (2013) 37133719.

[25] X. Yang, G. Zhu, S. Wang, R. Zhang, L. Lin, W. Wu, Z. L. Wang, Energy Environ. Sci. 5 (2012) $9462-$ 9466.

[26] Z.-H. Lin, G. Zhu, Y. Zhou, Y. Yang, P. Bai, J. Chen, Z. L. Wang, Angew. Chem. 19 (2013) 5065-5069.

[27] P. Bai, G. Zhu, Y. Liu, J. Chen, Q. Jing, W. Q. Yang, Z. L. Wang, ACS Nano. 7 (2013) 6361-6366.

[28] C. Zhang, T. Zhou, W. Tang, C. Han, L. Zhang, Z. L. Wang, Adv. Energy Mater. 4 (2014) 1301798.

[29] G. Cheng, Z.-H. Lin, L. Lin, Z. L. Du, Z. L. Wang, ACS Nano. 7 (2013) 7383-7391.

\section{Figure captions}



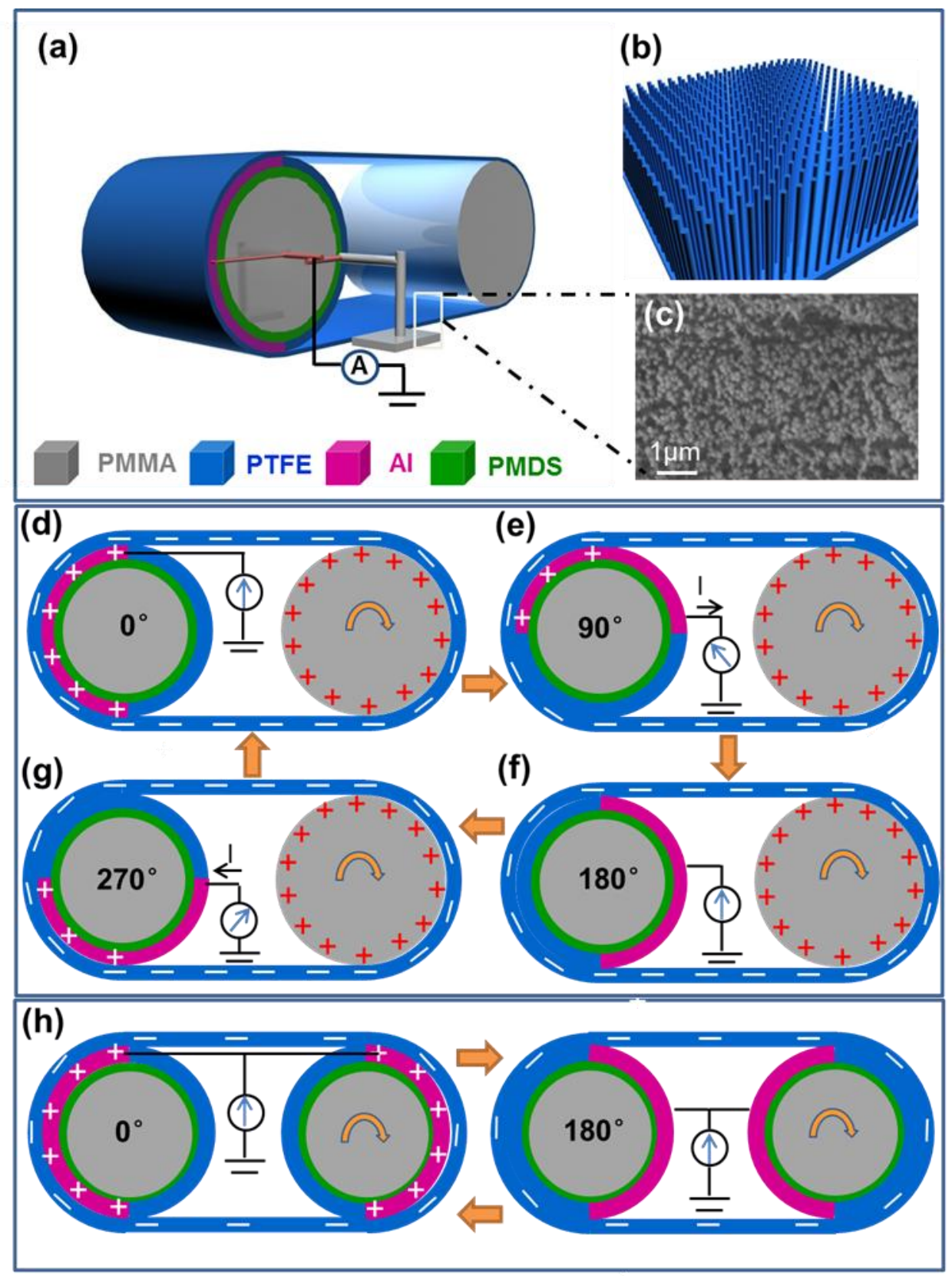

Figure 1. Basic structure and proposed working principle of the SR-TENG. (a) Schematic diagram. The structure diagram (b) and SEM image (c) of the PTFE film surface with nanostructure. (d)-(f) Schematic illustrations showing the working principle of the SR-TENG in four stages within a full cycle of electricity generation (The rotating angle of $\mathrm{Al}$ film is $0^{\circ}$, $90^{\circ}, 180^{\circ}$, and $270^{\circ}$, respectively). (h) The structure with two working wheels and its working principle. 
(a)

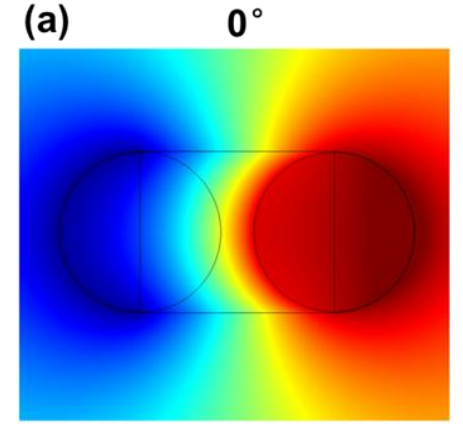

(d)



(g)

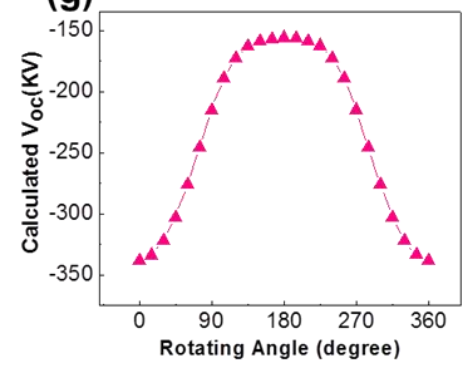

(b)

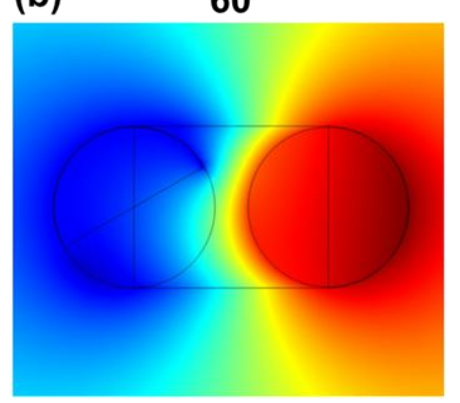

(e)

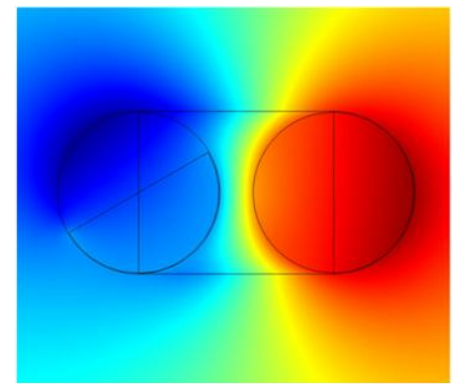

(h)

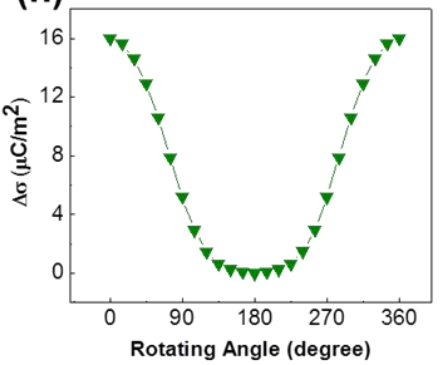

(c) $120^{\circ}$



(f) $300^{\circ}$
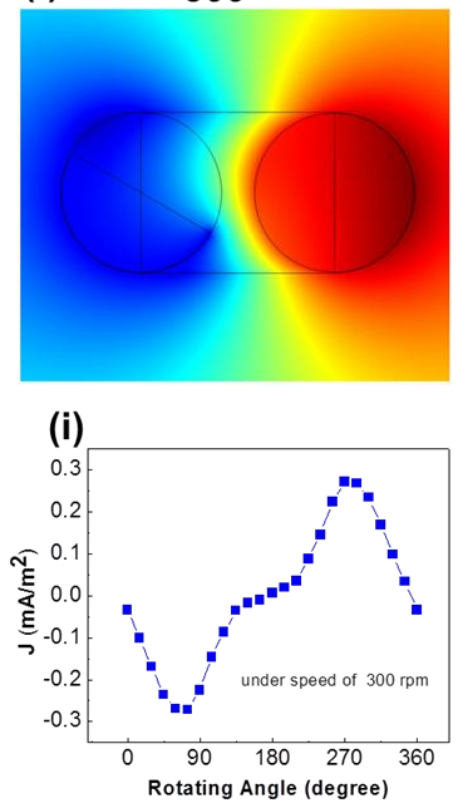

Figure 2. Finite element calculations of the SR-TENG. (a)-(f) Electric potential distribution within a full cycle of rotational motion (the rotating angle is $0^{\circ}, 60^{\circ}, 120^{\circ}, 180^{\circ}, 240^{\circ}$, and $300^{\circ}$, respectively). (g) - (i) The dependences of the calculated open-circuit voltage, transferred charge density and current density on rotating angle in a full cycle. The current density is calculated by setting the rotating speed as $300 \mathrm{rpm}$. 
(a)

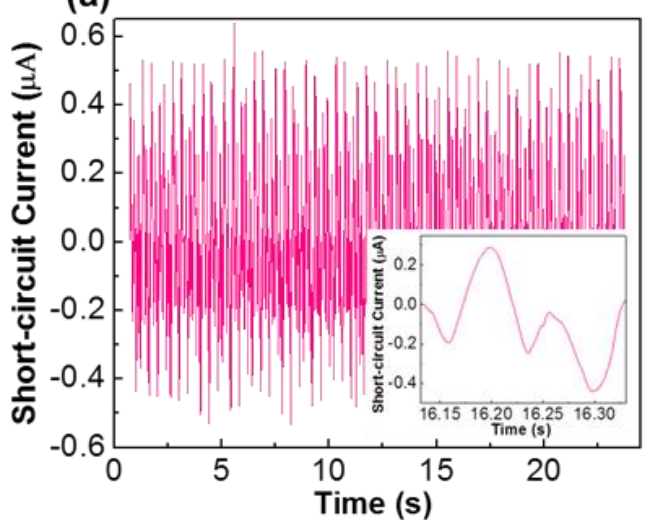

(c)

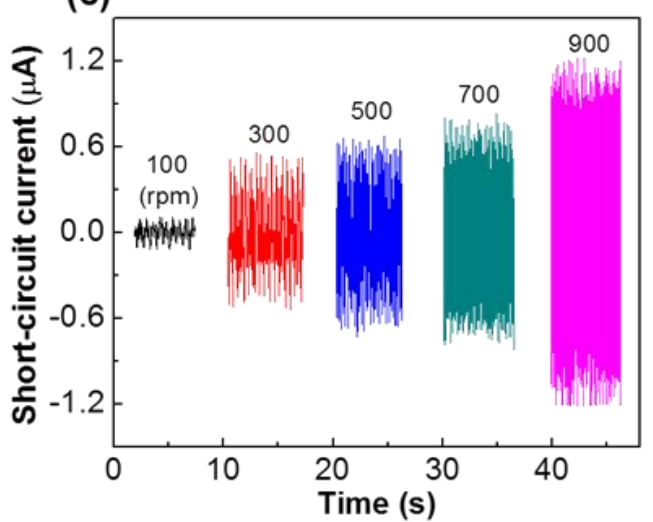

(e)

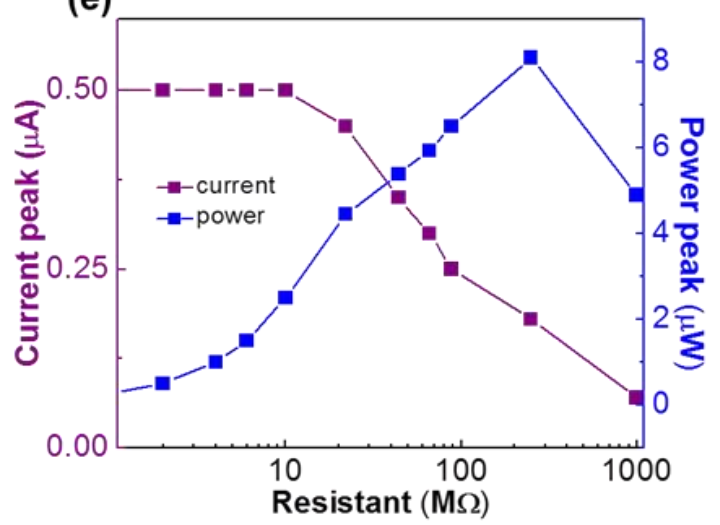

(b)

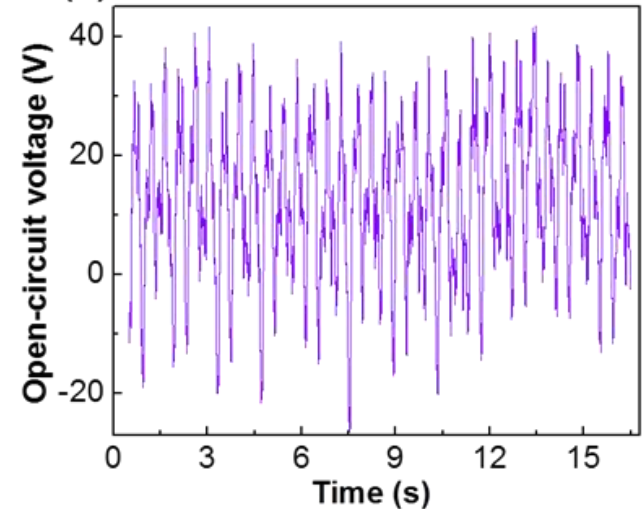

(d)

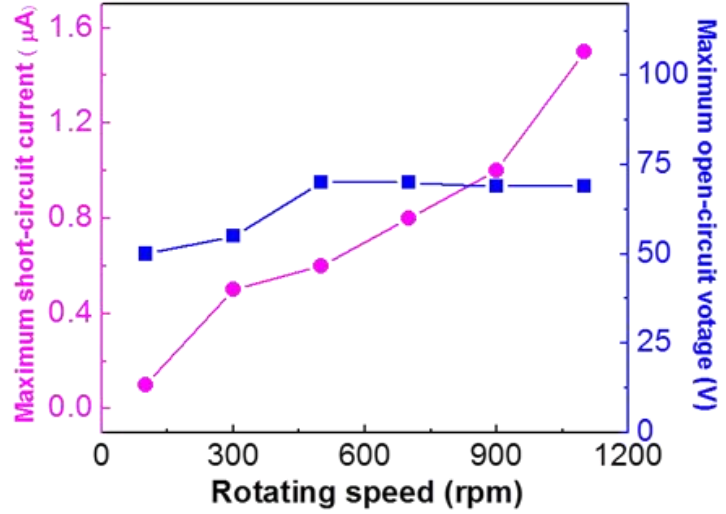

(f)

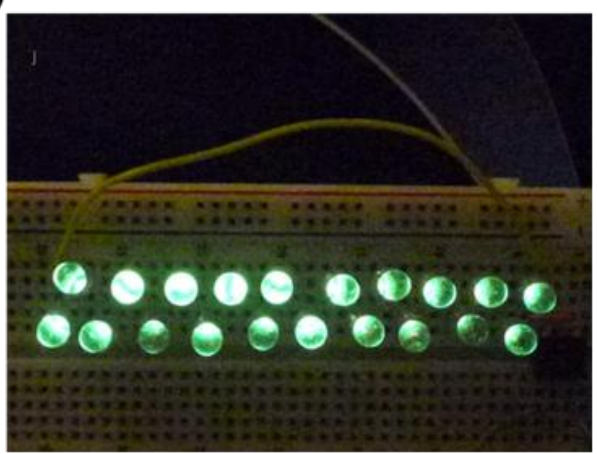

Figure 3. Output performance of the SR-TENG. (a) The measured short-circuit current at a rotating speed of $300 \mathrm{rpm}$. Inset: enlarged view of the current peaks. (b) The measured opencircuit voltage at a rotating speed of $300 \mathrm{rpm}$. (c) The measured short-circuit current curves with different rotating speed from 100 to $1100 \mathrm{rpm}$. (d) The summarized relationship between the currents/voltages and rotating speed. (e) The relationship between output current/power peaks and the resistance of an external load with a rotating speed of $500 \mathrm{rpm}$. (f) The 
photography of 20 commercial LED bulbs driven by the SR-TENG at a rotating speed of $1100 \mathrm{rpm}$.

(a)

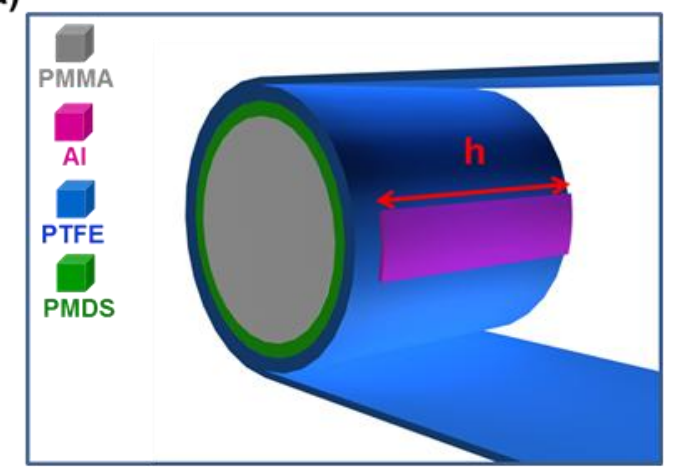

$h(\mathrm{~mm})$ (b)
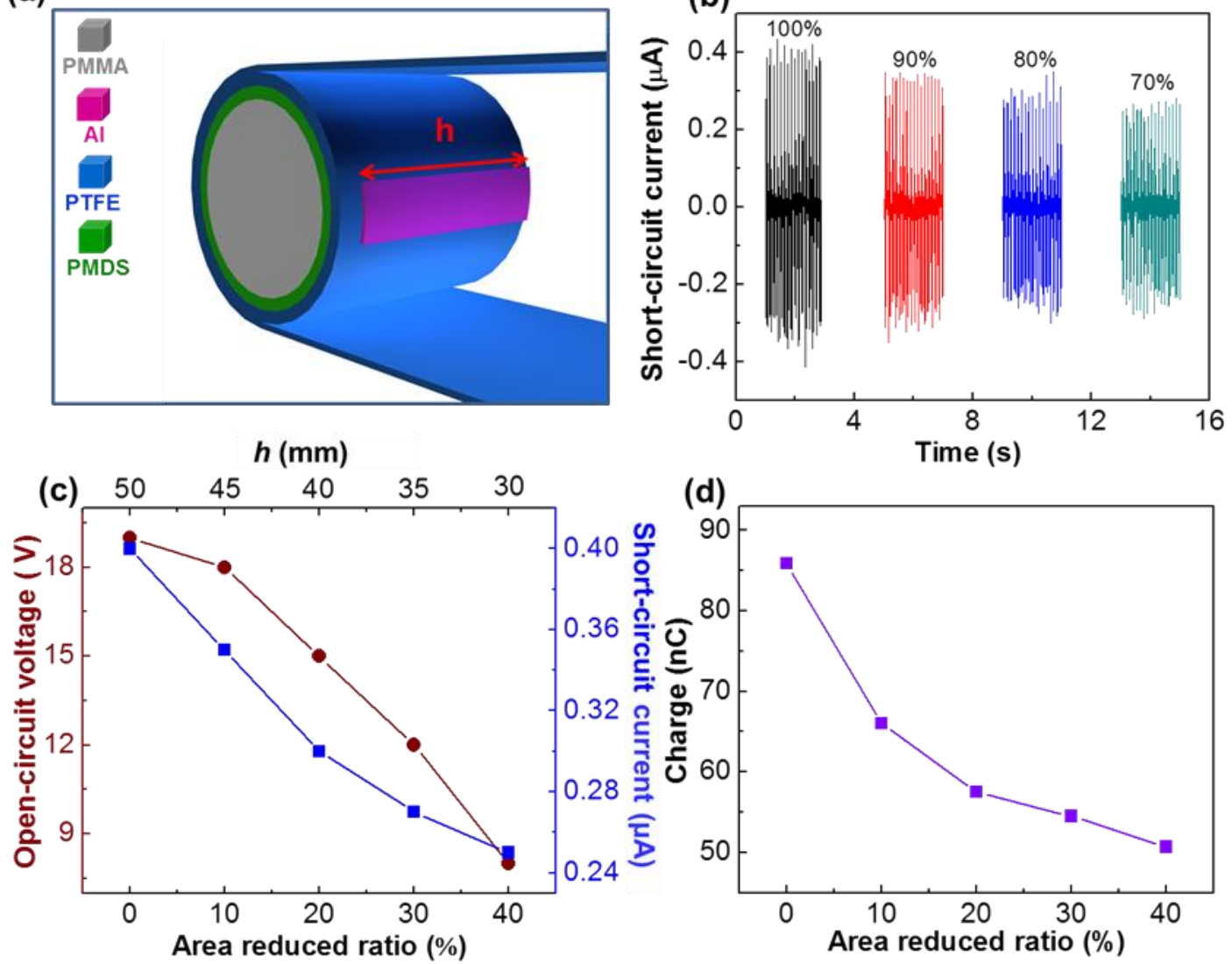

(d)

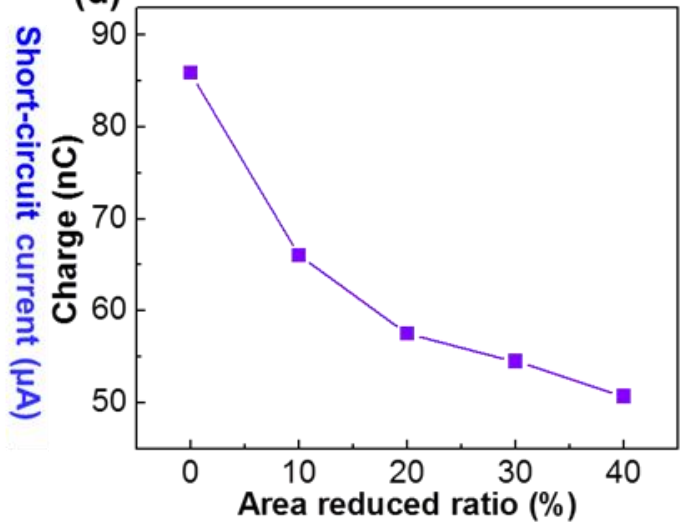

Figure 4. Influence of the $\mathrm{Al}$ foil area reduced ratio on the output performance of the SRTENG. (a) Schematic diagram. (b) The short-circuit current at a rotating speed of $500 \mathrm{rpm}$ with different area. (c) The dependence of the short-circuit current and the open-circuit voltage on area reduced ratio at a rotating speed of $500 \mathrm{rpm}$. (d) The dependence of transferred charge on area reduced ratio at a rotating speed of $500 \mathrm{rpm}$. 
(a)

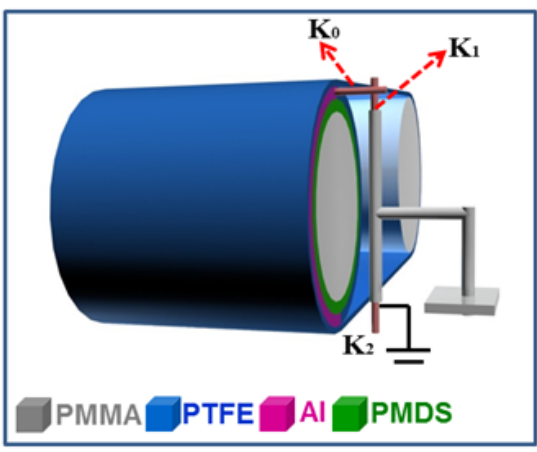

(f)

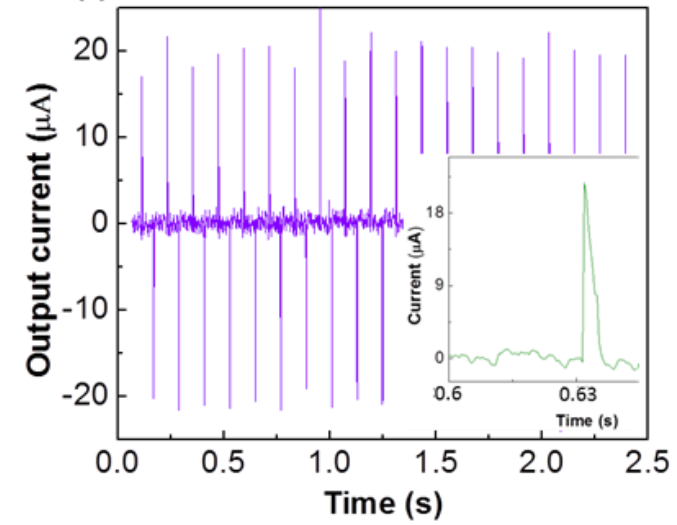

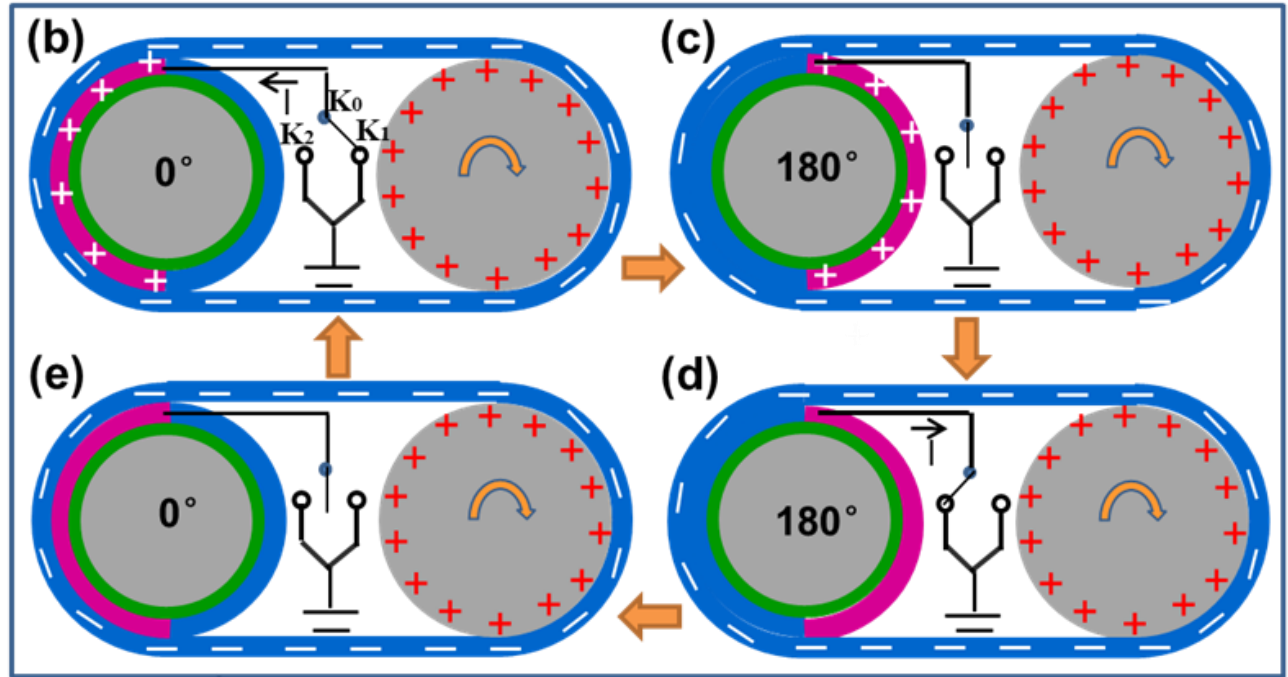
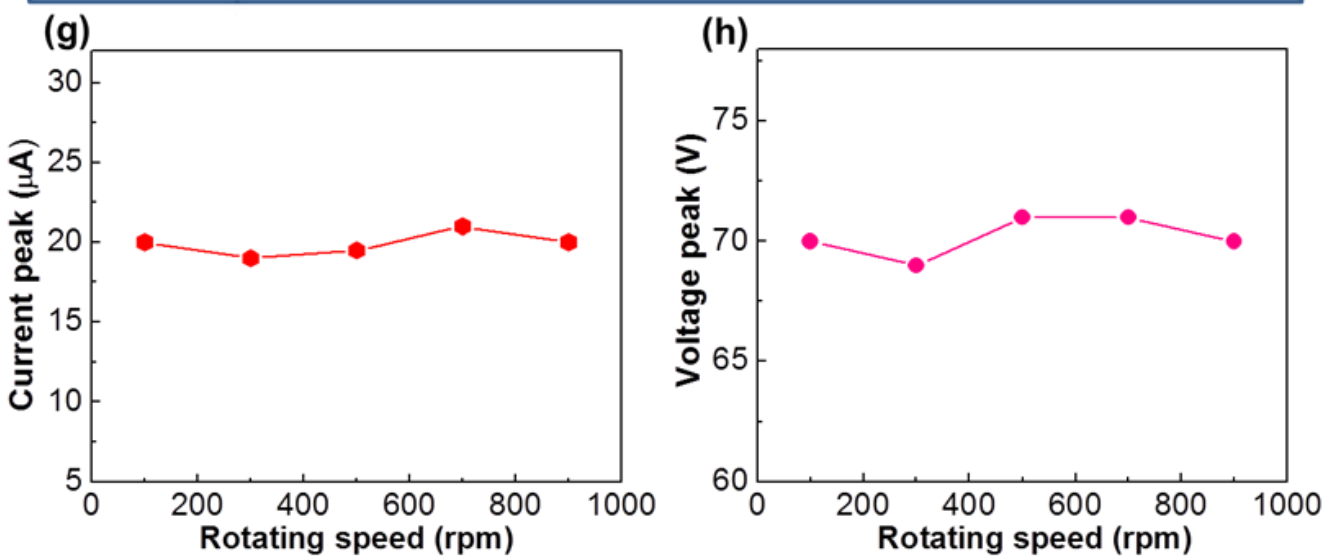

Figure 5. (a) Schematic diagram of the SR-TENG with ID mode. (b)-(e) Working mechanism of the SR-TENG with ID mode. (f) The output current at a rotating speed of $300 \mathrm{rpm}$ and a load of $10 \mathrm{M} \Omega$. Insert: magnification of a single current peak. The dependence of current peaks (g) and voltage peaks (h) on rotating speeds ranging from 100 to $900 \mathrm{rpm}$. 
(a)

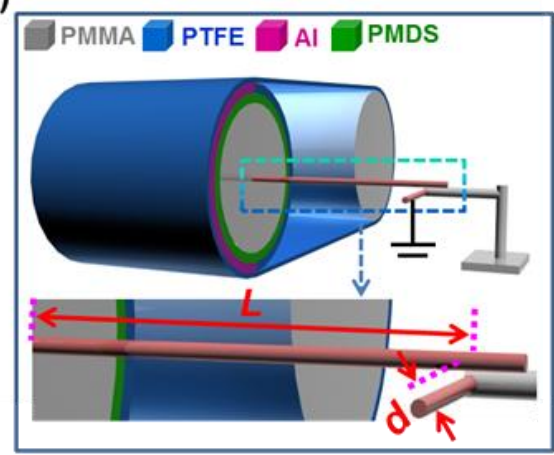

(c)

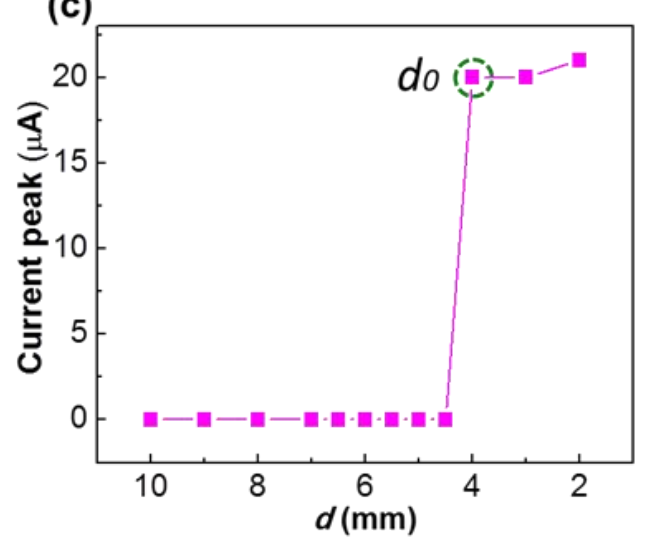

(b)

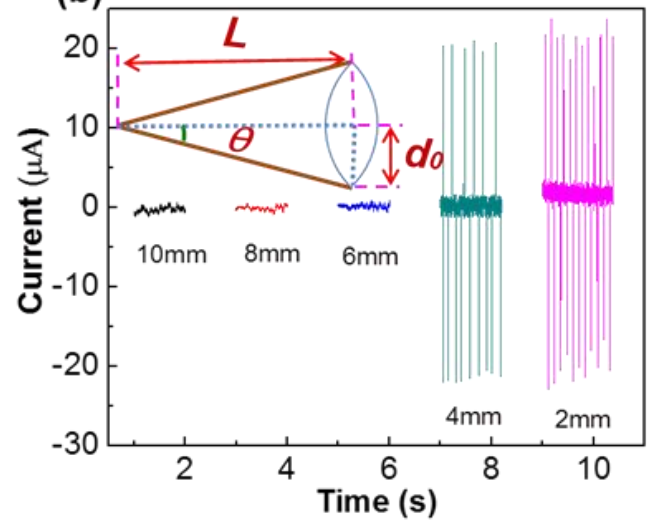

(d)

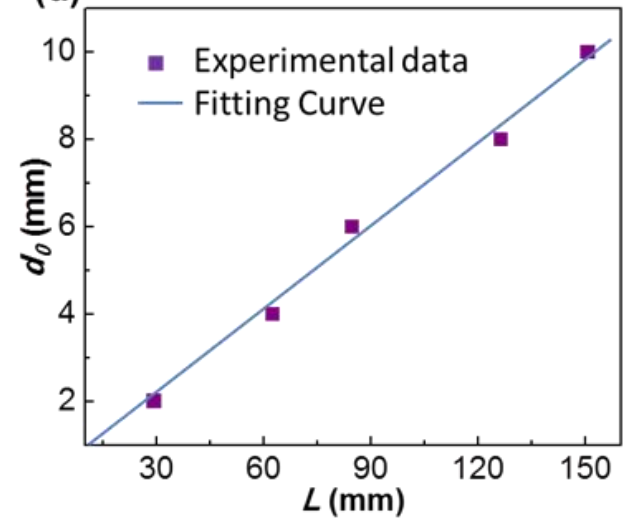

Figure 6. (a) The diagram of a misalignment sensor by using SR-TENG with ID mode. (b) The output current curves with different distances $(d)$ at a rotating speed of $500 \mathrm{rpm}$ and a load of $10 \mathrm{M} \Omega$. (c) The dependence of current peaks on distances. (d) The dependence of the critical distance $\left(d_{0}\right)$ on the length $(L)$, and the fitted straight line. 


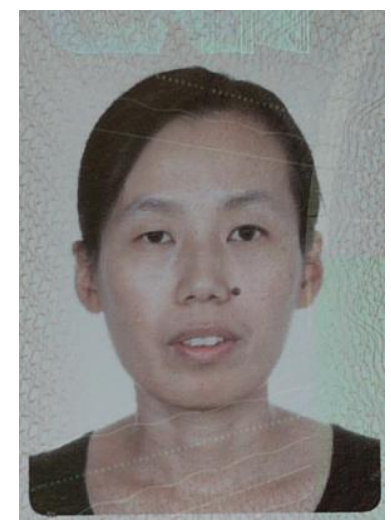

Yufang Li received her B. S. degree and Ph.D. degree in Institute of Metal Research from Chinese Academy of Sciences (CAS) in 2004. She is an associated professor in College of Materials Science and Technology of Nanjing University of Aeronautics and Astronautic (NUAA). Sis a visiting scholar in School of Materials Science and Engineering at Georgia Institute of Technology under the supervision of Prof. Zhong Lin (Z. L.) Wang. Her current research interests include triboelectric nanogenerator, nanostructured semiconductor and optoelectronic device.

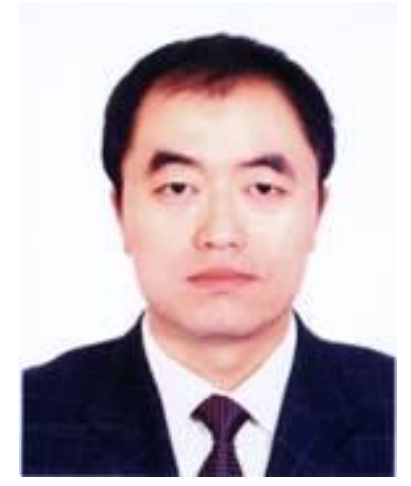

Gang Cheng is visiting scholar in School of Materials Science and Engineering at Georgia Institute of Technology and an associate professor in Henan University. He received the B. S. degree from Henan University in 2000 and the Ph. D. degree from Jilin University in 2008. His research interests are nanostructure-based electronic and optoelectronic devices, nanogenerator, and self-powered nanosensors. 


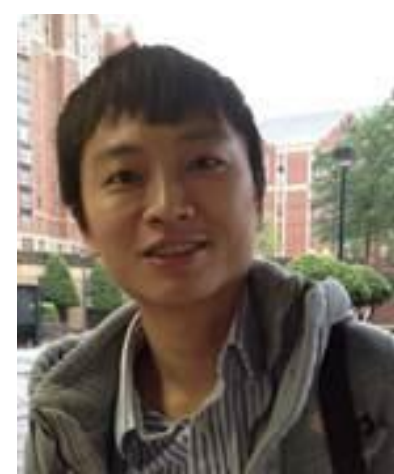

Zong-Hong Lin received his Ph.D degree from Department of Chemistry, National Taiwan Univerdity in 2009. Before joining the Institute of Biomedical Engineering, National Tsing Hua University in Taiwan as an assistant professor. He was a postdoctoral researcher supervised by Professor Zhong Lin Wang in the school of Materials Science and Engineering at Georgia Institute of Technology. His research interests include the development of highoutput generators with novel design for efficient energy harvesting, self-powered systems for biomolecules detection and environmental sensors, highely efficient and stable catalysts for fuel cell applications, and fabrication of metal miconductor nanowires

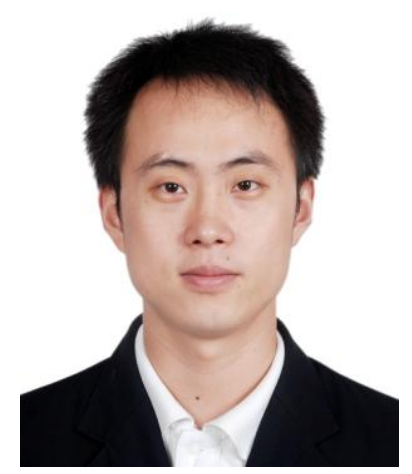

Jin Yang received the $\mathrm{BE}, \mathrm{ME}$ and $\mathrm{PhD}$ degrees in instrumentation science and technology from Chongqing University in 2002, 2004, and 2007, respectively. He is a professor with the College of Optoelectronic Engineering, Chongqing University. Currently, he works as a visiting researcher in School of Materials Science and Engineering at Georgia Institute of Technology under the supervision of Prof. Zhong Lin (Z. L.) Wang. His current research interests focus on sensor and actuator, measurement and instrumentation, nanogenerator, self-powered sensor and systems. 


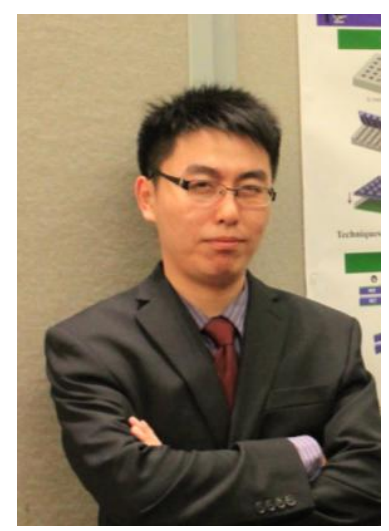

Long Lin received his B.S. in Materials Science and Engineering from Tsinghua University, China in 2010. He is currently a Ph.D. candidate in School of Materials Science and Engineering at Georgia Institute of Technology. He is working as a graduate research assistant at Prof. Zhong Lin Wang's group. His research mainly focuses on nanomaterial synthesis and characterization, devices for energy harvesting and storage, piezoelectric and triboelectric nanogenerators, self-powered nanosystems and active sensors, piezo-tronic and piezo-phototronic effect of semiconductor nanostructures and various applications.

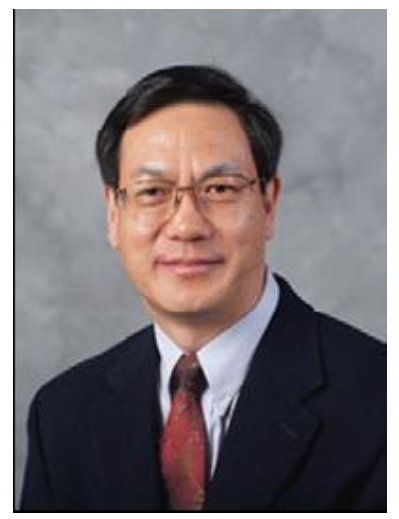

Zhong Lin (ZL) Wang received his Ph.D. from Arizona State University in physics. He now is the Hightower Chair in Materials Science and Engineering, Regents' Professor, Engineering Distinguished Professor and Director, Center for Nanostructure Characterization, at Georgia Tech. Dr. Wang has made original and innovative contributions to the synthesis, discovery, characterization and understanding of fundamental physical properties of oxide nanobelts and nanowires, as well as applications of nanowires in energy sciences, electronics, optoelectronics and biological science. His discovery and breakthroughs in developing nanogenerators established the principle and technological road map for harvesting mechanical energy from environment and biological systems for powering a personal electronics. His research on self-powered nanosystems has inspired the worldwide effort in academia and industry for studying energy for micro-nano-systems, which is now a distinct disciplinary in energy research and future sensor networks. He coined and pioneered the field of piezotronics and piezo-phototronics by introducing piezoelectric potential gated charge transport process in fabricating new electronic and optoelectronic devices. Details can be found at: http://www. nanoscience.gatech.edu. 


\begin{abstract}
We introduce a single-electrode-based rotationary triboelectric nanogenerator (SR-TENG) formed by two wheels and a belt for harvesting mechanical energy. The fundamental working principle is studied by conjunction of experimental results with finite element calculation. The continuous discharging (CD) mode and the instantaneous discharging (ID) mode have been demonstrated for the SR-TENG. The systematical experiments indicate that the short-circuit current increases with the rotating speed for SR-TENG with CD mode, but the open-circuit voltage maintains constant. The short-circuit current and open-circuit voltage decrease nearly linearly with the friction contact area, which provides an application as a self-powered surface area sensor of transmission wheel and gear. For SR-TENG with ID mode, the electric outputs are greatly enhances. The current peak is about $20 \mu \mathrm{A}$ at variation rotating speeds even if the external load is $10 \mathrm{M} \Omega$, which is 33 times higher than that of the SR-TENG with CD mode without external load. The SR-TENG with ID mode has also been demonstrated as a self-powered misalignment sensor.
\end{abstract}

Keywords: triboelectric nanogenerator, rotating energy, continuous discharge, instantaneous discharge, self-powered sensor

\title{
TOC Graphic:
}

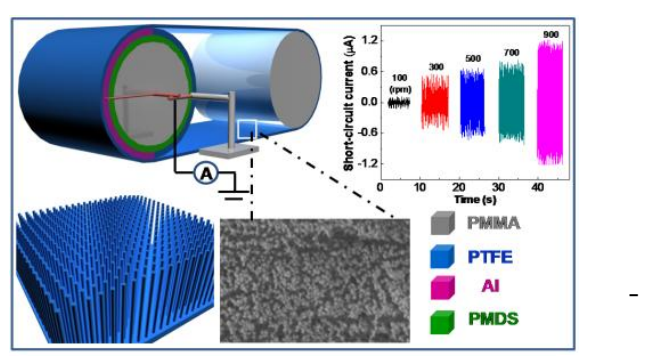

